Provided for non-commercial research and education use. Not for reproduction, distribution or commercial use.

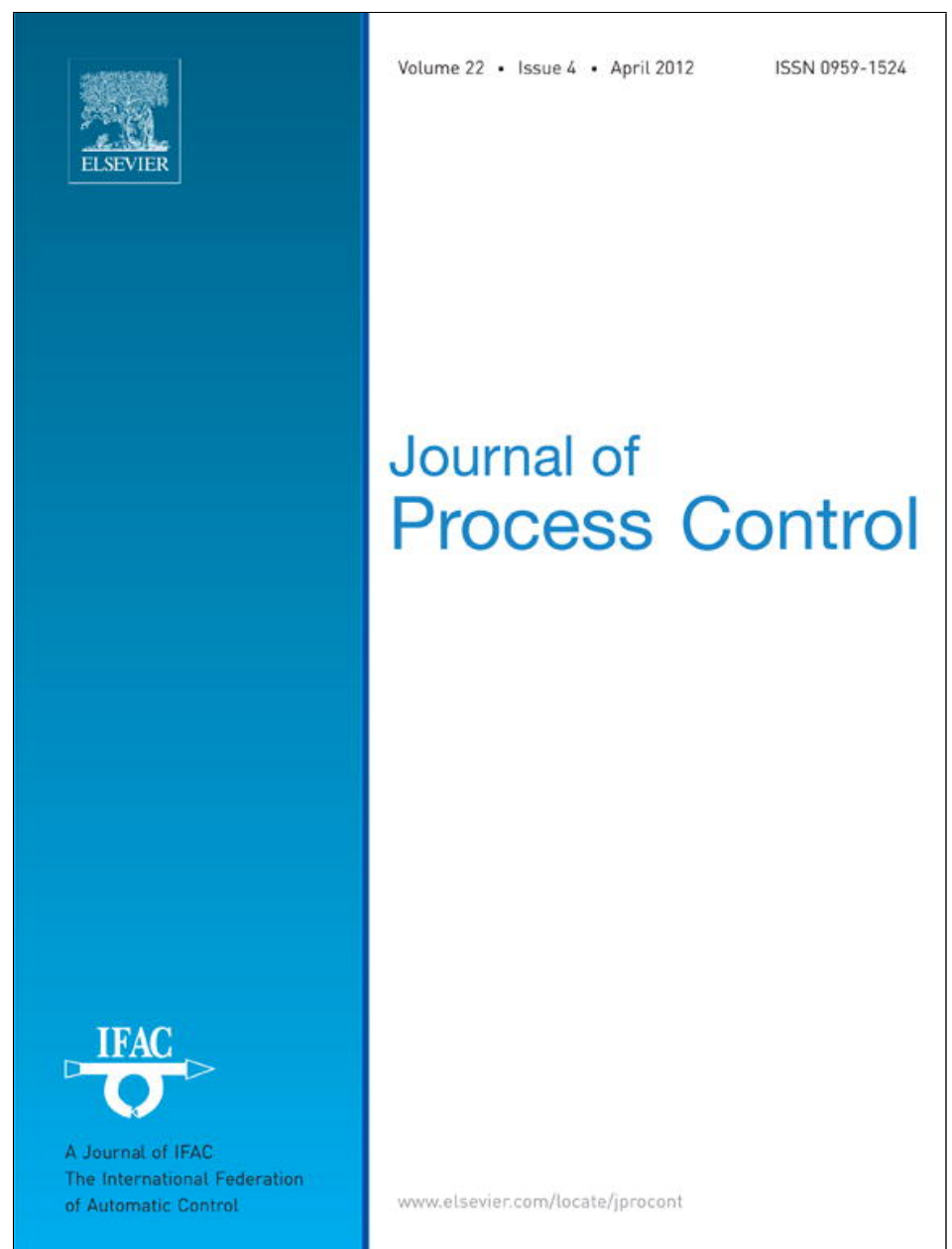

This article appeared in a journal published by Elsevier. The attached copy is furnished to the author for internal non-commercial research and education use, including for instruction at the authors institution and sharing with colleagues.

Other uses, including reproduction and distribution, or selling or licensing copies, or posting to personal, institutional or third party websites are prohibited.

In most cases authors are permitted to post their version of the article (e.g. in Word or Tex form) to their personal website or institutional repository. Authors requiring further information regarding Elsevier's archiving and manuscript policies are encouraged to visit:

http://www.elsevier.com/copyright 


\title{
Real-time virtual metrology and control for plasma etch
}

\author{
Shane A. Lynn ${ }^{\mathrm{a}, *}$, Niall MacGearailt ${ }^{\mathrm{b}}$, John V. Ringwood ${ }^{\mathrm{a}}$ \\ a Dynamics and Control Group, Electronic Engineering Department, National University of Ireland, Maynooth, Ireland ${ }^{1}$ \\ ${ }^{\mathrm{b}}$ Intel Ireland Ltd., Collinstown, Leixlip, Co. Kildare, Ireland
}

\section{A R T I C L E I N F O}

\section{Article history:}

Received 23 June 2011

Received in revised form 8 December 2011

Accepted 19 January 2012

Available online 13 March 2012

\section{Keywords:}

Virtual metrology

Plasma etch

Predictive functional control

Electron density

Etch rate

Advanced process control

\begin{abstract}
A B S T R A C T
Plasma etch is a semiconductor manufacturing process during which material is removed from the surface of semiconducting wafers, typically made of silicon, using gases in plasma form. A host of chemical and electrical complexities make the etch process notoriously difficult to model and troublesome to control. This work demonstrates the use of a real-time model predictive control scheme to control plasma electron density and plasma etch rate in the presence of disturbances to the ground path of the chamber. Virtual metrology (VM) models, using plasma impedance measurements, are used to estimate the plasma electron density and plasma etch rate in real time for control, eliminating the requirement for invasive measurements. The virtual metrology and control schemes exhibit fast set-point tracking and disturbance rejection capabilities. Etch rate can be controlled to within $1 \%$ of the desired value. Such control represents a significant improvement over open-loop operation of etch tools, where variances in etch rate of up to $5 \%$ can be observed during production processes due to disturbances in tool state and material properties.
\end{abstract}

(C) 2012 Elsevier Ltd. All rights reserved.

\section{Introduction}

High-volume, high-yield, and high-throughput manufacturing is of primary importance in modern semiconductor manufacturing. Product wafers in a semiconductor manufacturing cycle typically undergo over 350 different process steps in their path from raw silicon wafer to finished product. The exponential increase in microprocessor capabilities predicted by Moore [1] has become a benchmark and target for semiconductor manufacturers, continually driving increases in performance with decreasing dimensions. Current industrial development is working towards manufacturing at the $22 \mathrm{~nm}$ node (half the distance between cells in a dynamic random access memory chip) in the near future [2].

In a semiconductor fabrication environment (or $f a b$ ), nanometer scale devices are constructed on the surface of silicon wafers through repetitive applications of five main processes; namely, deposition, lithography, etch, ion implantation, and planarisation. Interdependencies exist between the processes and tight control of each manufacturing process is essential to reduce defects that can reduce the product yield (the fraction of functional devices produced on the finished wafers).

Plasma etch is a key process in the manufacturing cycle. During plasma etch, etchant gases in plasma form are directed towards the

\footnotetext{
* Corresponding author.

E-mail address: shane.a.lynn@eeng.nuim.ie (S.A. Lynn).

1 Tel.: +35317084766.
}

wafer surface using electric and magnetic fields. The gases react with the exposed areas of the wafer surface, and the etch product subsequently evaporates to remove material. Plasma etching is preferred to wet etching methods (using etchant in liquid phase) because it is capable of producing a highly anisotropic etch, allowing deep and narrow trenches to be etched in the wafer surface [3]. Such etch profiles are desirable for tight packing of components on the wafer surface.

Plasma etch is conducted within specialised etch chambers. Process input variables to the chambers are typically well controlled variables such as chamber pressures, component temperatures, and gas flow rates that are specified by set points. In general, the required etch process input variables for each product are developed through extensive experimentation during the product development stage, early in a product's life cycle. Once decided upon, the etch process input variables are compiled into recipes that are distributed to different fabs for production. The etch recipes remain relatively fixed and, typically, the recipes are applied to product wafers in each fab environment in an open-loop manner [4]. This open-loop application of process recipes is still predominantly used for plasma etch processes.

Achieving consistent etch performance with open-loop control is difficult as a result of the time-varying nature of the plasma etch process. Etch processes exhibit process drift and unpredictable shifts in behaviour due to chamber conditioning, incoming wafer variability, and the unpredictable effects of chamber maintenance activities. It is important to control and monitor the etch rate as precisely as possible since the ultimate etch depth and profile 


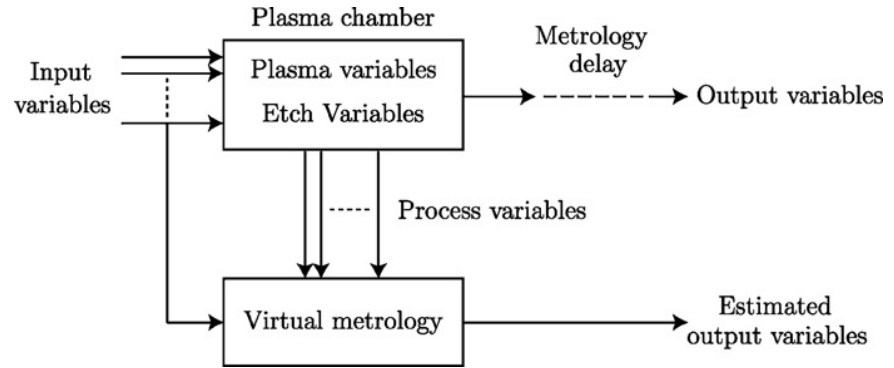

Fig. 1. Virtual metrology principle. Estimates of process output variables of interest are made using process variables and mathematical models, or virtual metrology models.

have a significant impact on the performance of the devices being produced. In situ measurements of etch rate and etch depth are expensive, time consuming, and often invasive. Measurements are not available to machine operators without a considerable metrology delay and it is not practical for fabrication plants to measure every wafer processed. Due to a relatively high throughput, a significant quantity of wafer scrap can result if a tool operates out of specification undetected. In an industry with high-value product material, such excursions can cost manufacturers thousands of dollars in revenue.

Plasma etch processes are predominantly managed using statistical process control (SPC) [5], where variables measured in situ during each process, or variables concerning the result of each process, are monitored for deviations that indicate erroneous operation. However, with SPC, metrology delays can lead to wafers being processed erroneously, and the process recipe still does not take the typical time-varying nature of the process into account.

Advanced process control (APC) and virtual metrology (VM) are enabling technologies that can resolve the control issues in semiconductor manufacturing [6]. Virtual metrology is the estimation of process metrology variables that may be expensive or difficult to measure using readily available process information. The process flow for a VM implementation is shown in Fig. 1. Fab-wide VM schemes that are capable of increasing factory throughput, reducing wafer scraps, cutting production costs, and paving the way to fully automated wafer-2-wafer (W2W) control have been investigated $[7,6,8]$.

The ultimate aim of APC is to improve device yield. APC is considered to include fault detection, fault classification, fault prognosis, and process control, using information about the material to be processed, measured data, and the desired results [9]. APC includes lot-to-lot, wafer-to-wafer, and within-wafer real-time control and can improve performance, yield, and throughput [10]. Thus far, APC implementation in the semiconductor industry has broadly been restricted to lot-to-lot control [7] because of infrequent measurements and large metrology delays. VM is a potential solution to overcome these difficulties. A considerable amount of research has been completed in the area of VM for plasma etch [4], with most effort being focussed on wafer-level estimation of process output variables such as etch rate or etch depth.

A number of possible control strategies using VM are depicted in Fig. 2. Plasma variables such as species concentrations and temperatures can be controlled in real-time, etch process variables such as etch rate (the rate of material removal from the wafer surface) can be controlled in real-time or on a wafer-to-wafer basis, and process output variables such as etch depth can be controlled on a wafer-to-wafer or lot-to-lot basis.

This paper focuses on real-time control of plasma electron density and plasma etch rate. Plasma electron density is known to be a key variable affecting etch process performance [11] and control of electron density during plasma etching has benefits for reduction of process variability, including reduction of the first-wafer effect $[12,13]$. However, for control, real-time measurement of plasma electron density is typically required using sensors that require permanent adaptations to the etch chamber to provide feedback for control. Imai [14] demonstrates the feasibility of using etch process variables for VM of electron density for fault detection purposes. In this paper, VM is investigated as a solution for feedback control.

Plasma etch rate is typically regulated using run-to-run control schemes, potentially using VM for feedback $[15,16]$. Real-time control of plasma etch rate has been reported by a number of researchers, but typically requires the use of bulky, expensive, or

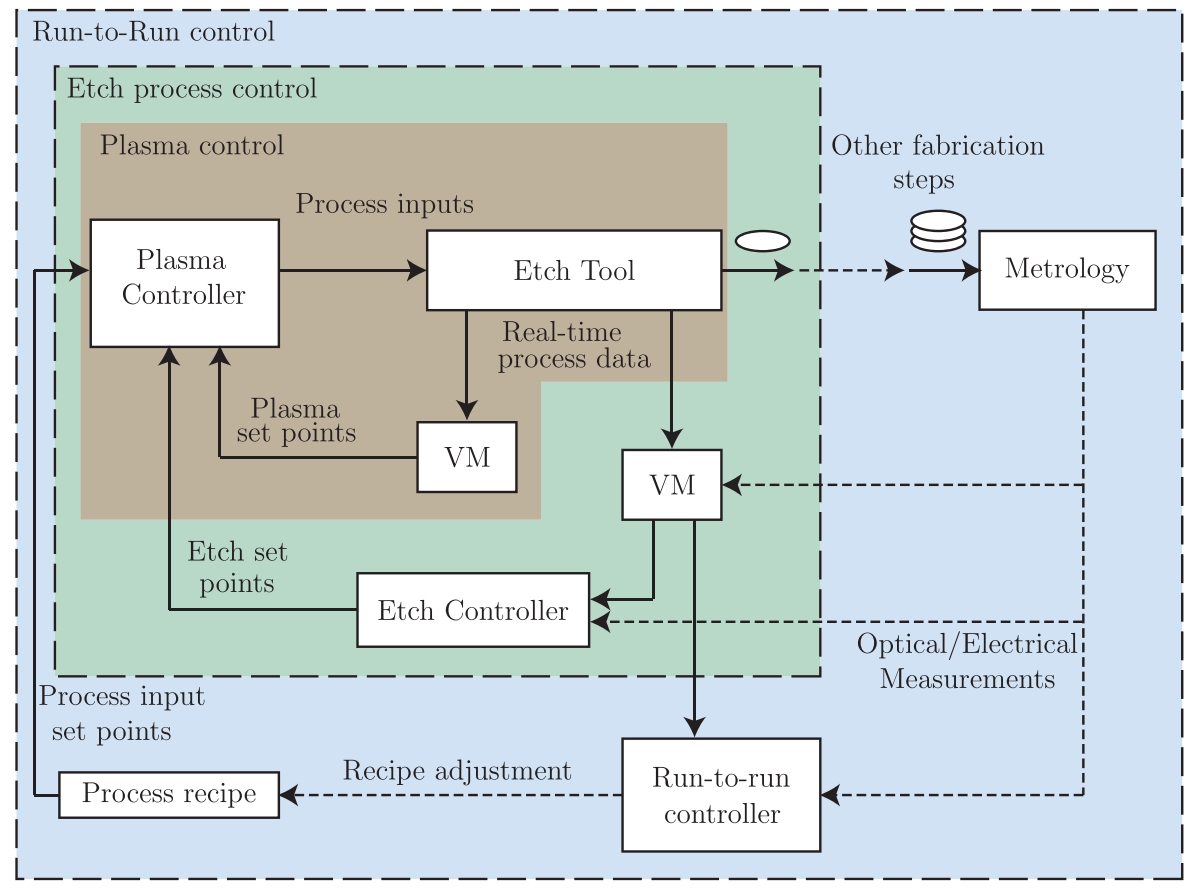

Fig. 2. Virtual metrology and control implementations for plasma etch [4]. 


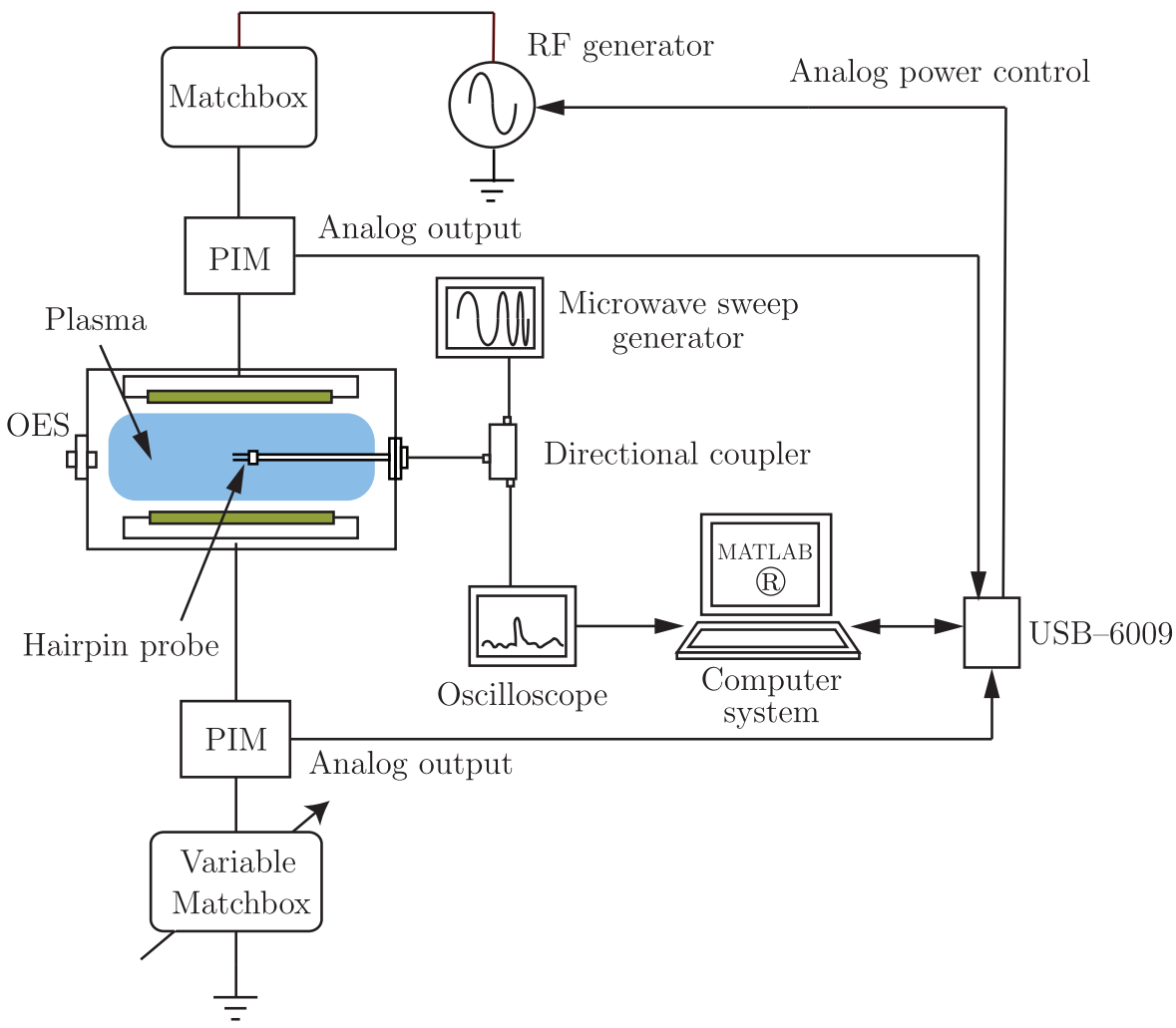

Fig. 3. Experimental apparatus for real-time VM and control of electron density and plasma etch rate.

invasive measurement techniques. For example, Sarfaty et al. [17] implement real-time control of etch rate using a proportionalintegral-derivative (PID) controller, model-based feed-forward action for large changes in etch rate set point, and laser reflectance interferometry (LRI) to measure the etch rate. Stokes and May $[18,19]$ describe control of etch rate using indirect adaptive control and data from LRI Laser interferometry (LI), residual gas analysis (RGA), and optical emission spectroscopy (OES) for process feedback. Rosen et al. [20] develop real-time feed-back controllers for etch processes based on in situ spectroscopic ellipsometry measurements of wafer thickness.

This work investigates the feasibility of real-time VM, using plasma impedance monitor (PIM) data, for real-time control of electron density and plasma etch rate. The benefits of the VM scheme is that the measurements are obtained non-invasively, allowing costeffective, real-time control without process perturbation. Control of both electron density and etch rate is achieved through the use of a model-based predictive control (MPC) scheme using applied RF power as the manipulated variable. Control is implemented using predictive functional control (PFC), an MPC technique.

Preventative maintenance (PM) events have a large influence on etch performance and VM model estimation accuracy. PM events involve the routine replacement of components, such as electrodes and ceramic covers, that have been exposed to etchant chemicals for possibly over 1000 wafer etch and cleaning cycles. Although the replacement components are macroscopically identical to those that are removed from the chamber, microscopic differences in the electrical connections made between components when they are replaced change the electrical characteristics of the chamber. Changes in such component connections are more influential as the applied RF frequency increases [21]. At the high frequencies in use during plasma processing $(\sim 13.56 \mathrm{MHz})$, changes in impedance, stray capacitances, and stray inductances cause considerable changes to the electrical behaviour of the chamber and hence the etching plasma properties. The electrical path between the powered chamber electrode and ground (the ground path) influences plasma variables such as the ion flux to the etching wafer and the DC bias of the wafer in the chamber [22]. Hence, changes in the impedance of the ground path brought about by PM events can cause the etch performance of the chamber to vary dramatically across maintenance cycle events.

For the experiments described in this paper, an extra modified match box that allows manual control of impedance is installed on the ground path from the chamber. Hence, variations in the ground impedance can be realised as required, partially simulating the effect of PM events. The ground impedance variations act as unmeasured disturbances to the plasma, changing plasma variables such as electron density, and affecting the etch performance.

The remainder of the paper is laid out as follows: Section 2 describes the experimental apparatus used. Section 3 provides the theoretical background for PFC. Section 4 describes the development of VM models for electron density and etch rate. Finally, Sections 5 and 6 give the control results and paper conclusions, respectively.

\section{Experimental setup}

An overview of the experimental setup is shown in Fig. 3 and each component is discussed briefly in the following subsections.

\subsection{Plasma etch chamber}

Plasma is generated in a capacitively coupled, top-powered, parallel-plate plasma etch chamber. Between 0 and $625 \mathrm{~W}$ of RF power at $13.56 \mathrm{MHz}$ is delivered to the topmost chamber electrode from an RF generator. The amount of delivered power is specified via a $0-10 \mathrm{~V}$ reference signal generated using a control computer using DAC hardware. Chamber pressure is controlled to a specified set point by means of a gate valve between the etch chamber and a vacuum turbo pump. 


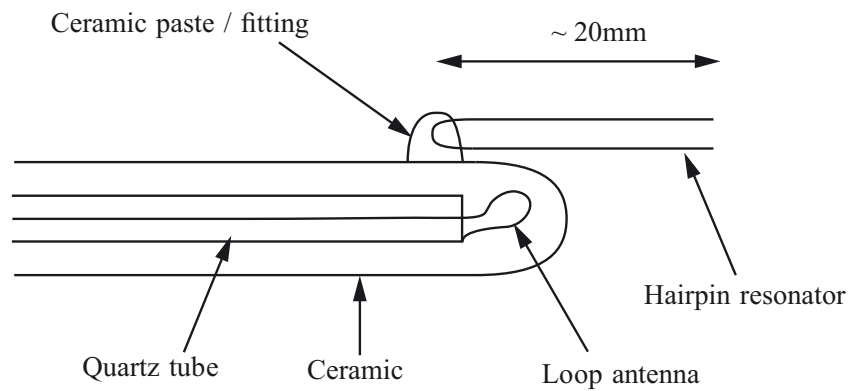

Fig. 4. Microwave resonator hairpin probe.

The bottom electrode in the etch chamber is grounded through a modified match unit, such that the position of the matching inductor can be varied manually, effecting a total ground impedance of between 0 and $70 \Omega$. Variations in this path act as disturbance signal to the plasma in the chamber. The plasma undergoes a mode change at approximately $25 \Omega$, above which etching at the wafer surface stops. Hence, for the experiments described here, the ground impedance is limited to a ceiling of $25 \Omega$. This range of ground impedance encapsulates those changes that typically occur as a result of PM events.

\subsection{PIM sensors}

A plasma impedance monitor (PIM) is an electronic sensor that is installed between the matching network and the plasma electrodes. The PIM sensor provides information on the current, voltage and phase of the waveforms on the power supply circuitry. Information on the fundamental frequency of $13.56 \mathrm{MHz}$ and up to 52 harmonics of this frequency is recorded. Power at the harmonic frequencies of the supplied power is generated in the supply circuitry as a result of the non-linear impedance presented by the oscillating plasma sheaths at the electrodes in the plasma chamber. Calculations of impedance, reactance, resistance, and power can be made from the PIM signals.

Two PIM sensors are used. One PIM is installed on the powered electrode of the chamber, and provides information on the applied $\mathrm{RF}$ power. The second sensor records information about the path to ground from the chamber.

Digital outputs from the PIM sensors are encoded using a proprietary format and are not available for use in real-time. However, analogue output channels on each PIM sensor provide real-time measurements, which can be used for control. Unfortunately, the analogue signals undergo a fixed delay of $0.5 \mathrm{~s}$ as a result of the internal circuitry and ADC used by the PIM processing units.

\subsection{Hairpin resonator probe}

The electron density in the plasma etch chamber is determined using a microwave hairpin resonator, termed a hairpin probe. Introduced by Stenzel [23] in the mid 1970s, a hairpin probe is an open-ended quarter wavelength transmission line whose resonant frequency is related the dielectric constant of the medium that surrounds it. Fig. 4 shows a schematic of a hairpin probe.

Energy is coupled into the U-shaped structure and, at resonance, a standing wave occurs on the hairpin such that the voltage is a maximum at the open end and a minimum at the shorted end of the transmission line. At resonance, the hairpin weakly radiates energy into the surrounding space whereas, at off-resonance, almost all energy incident from the current source is reflected [24].
The plasma electron density is related to the frequency difference between the hairpin resonances with and without the plasma,

$n_{e}=\frac{f_{r}^{2}-f_{0}^{2}}{0.81}$,

where $n_{e} \times 10^{10} \mathrm{~cm}^{-3}$ is the electron density, and $f_{r}$ and $f_{0}$ are the resonant frequencies (in gigahertz) of the hairpin with and without the plasma, respectively [25].

\subsection{Data acquisition and control system}

A control computer manages the data flow between all of the actuator and sensors in the system. All of the control calculations are performed using the MATLAB ${ }^{\circledR}$ programming environment. The VM input variables are measured using a National Instruments USB6009 ADC connected to the PIM sensors, and can be sampled at a higher rate than the electron density probe. For the experiments in this work, the analogue sampling rate (and hence the VM measurement rate) is set to $10 \mathrm{~Hz}$ to allow for noise averaging during each sample.

\subsection{Validation measurements}

For etching experiments, $200 \mathrm{~mm}$ diameter silicon wafers coated with a thin layer (approximately $6000 \AA$ ) of polysilicon are used. To measure the true average etch rate of the process, the thickness of the polysilicon layer is measured precisely using an interferometer at 19 different locations on the wafer surface both before and after wafer processing. The mean depth change over all of the measured locations is recorded as the etch depth achieved for the wafer. The average etch rate is calculated by dividing the etch depth by the known etch time. In situ measurements of etch rate or etch depth are not available for the process under study.

\section{Predictive functional control}

\subsection{Motivation}

Model predictive control (MPC) or model-based predictive control was first employed in the 1970s in the defence and petroleum industries. Predictive functional control (PFC) is differentiated from the other forms of MPC in that the internal models used are independent internal models that depend solely on the process input. Furthermore, the manipulated variable is constructed on a set of basis functions, typically a polynomial basis [26]. PFC is chosen as the MPC variant for the control of electron density in the plasma etch chamber for a number of reasons: It is easily implemented using a first order approximation to the system, it uses a single intuitively interpreted parameter during tuning, it is designed primarily for single-input single-output systems, and it can control the etch system taking the VM delay into account. A closed-form solution can be derived for a first-order PFC implementation, negating the requirement for online optimisation during control operations.

\subsection{Internal model}

The "internal model" is a model of the plant used by a predictive controller that is capable of predicting future process outputs. The internal model is not restricted to a particular form and can be formulated as a transfer function, state-space, step-response, black-box model, etc. Consider a zero-order hold equivalent model for a first-order process, having a gain $K_{m}$ and a time constant $\tau_{m}$ as

$y_{m}(k)=a_{m} y_{m}(k-1)+b_{m} K_{m} u(k-1)$, 


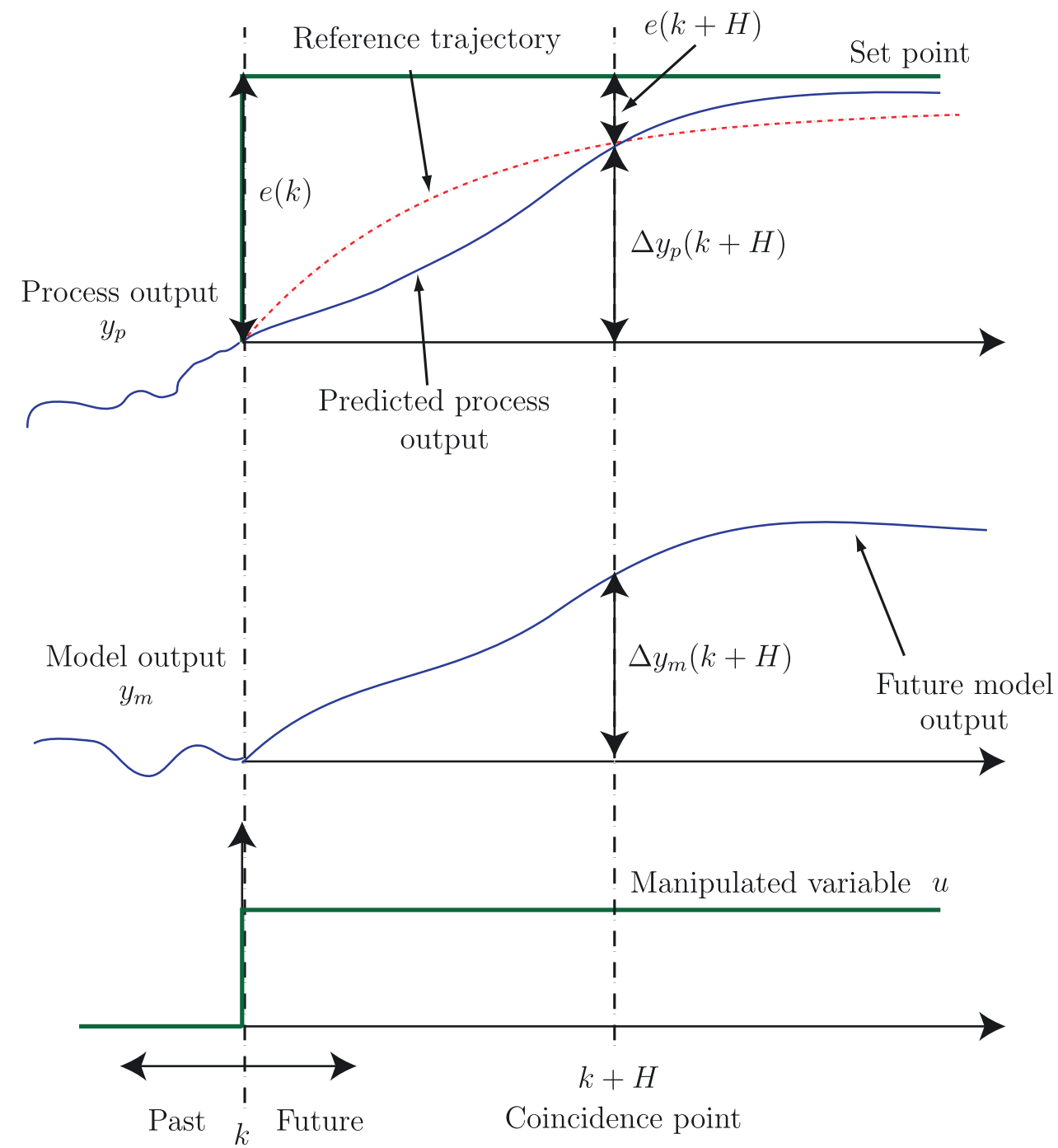

Fig. 5. Reference trajectory, model increment, and process increment in PFC.

where $a_{m}=e^{-T_{s} / \tau_{m}}, b_{m}=1-a_{m}$, and $T_{s}$ is the system sample period. The actual process output is $y_{p}$. Eq. (2) describes an independent model that calculates the output $y_{m}$ using only the known measured process inputs and past model outputs. Because the process may be subjected to unknown disturbances and the plant model will not be perfect, $y_{p} \neq y_{m}$. However, $y_{p}$ and $y_{m}$ will evolve in parallel, and the model is used to calculate increments of the process output rather than the absolute response of the process subjected to a particular input [26].

The model prediction of the process response to a step change in input, from the instant $k=0$ to a future time $k+H$, where $H$ is an integer number of samples, consists of the free solution $y(k) a_{m}^{H}$ and the forced solution $K_{m} u(k)\left(1-a_{m}^{H}\right)$. By superposition, the full solution to $y(k+H)$ consists of the sum of the free and the forced responses.

\subsection{Reference trajectory}

The desired future behaviour of the controlled variable is the "reference trajectory". The reference trajectory is initialised on the current process output $y_{p}(k)$, and defines the path taken by the controlled variable to the current set point $S(k)$.

The "coincidence horizon" is the set of points in the future where the process and the model outputs should be equal. For the sake of simplicity, only one coincidence point $H$ is considered. Typically, an exponential reference trajectory is defined such that the error signal at a time $k+H$ is

$S-y_{p}(k+H)=e(k+H)=e(k) \lambda^{H}$,

where $S$ is a constant set point, $\lambda=e^{-T_{s} / \tau_{r}}$, with $\tau_{r}$ the desired closedloop time constant of the controlled system.

As evident from Fig. 5, the desired process output increment at the coincidence point, $\Delta y_{p}(k+H)$, is given by

$\Delta y_{p}(k+H)=e(k)-e(k+H)$.

Hence

$\Delta y_{p}(k+H)=-e(k) \lambda^{H}+e(k)=\left(S-y_{p}(k)\right)\left(1-\lambda^{H}\right)$

At each sample time $k$, the values for $\Delta y_{p}$ are computed, and the first value is applied to the plant and model. At the next sample time, $k+1$, the procedure is repeated, resulting in a new reference trajectory, in essence creating a receding horizon. 


\subsection{Calculation of controlled variable}

The future manipulated variable $u(k)$ is structured around a set of basis functions that are chosen according to the nature of the process and set point variations:

$u(k+i)=\sum_{j=0}^{N-1} \mu_{j} F_{j}(i), \quad 0 \leq i \leq H$,

where $F_{0}, F_{1}, \cdots, F_{N-1}$ are the basis functions, and $\mu_{0}, \mu_{1}, \cdots, \mu_{N-1}$ are the weights associated with the functions. Thus, the manipulated variable is expressed as a weighted sum of $N$ basis functions. PFC generally uses a set of polynomial basis functions, i.e., $F_{j}(i)=i^{i}$. In the elementary case, including the case that applies here, the basis functions reduce to $N=1, F_{0}(i)=i^{0}=1$.

As seen before, $\Delta y_{p}(k+H)=\left(S-y_{p}(k)\right)\left(1-\lambda^{H}\right) . \quad \Delta y_{m}(k+H)$, where $\Delta y_{m}$ is the model increment, is given by

$\Delta y_{m}(k+H)=y_{m}(k+H)-y_{m}(k)$,

$\Delta y_{m}(k+H)=y_{m}(k) a_{m}^{H}+K_{m} u(k)\left(1-a_{m}^{H}\right)-y_{m}(k)$.

The equality $\Delta y_{p}(k+H)=\Delta y_{m}(k+H)$ is achieved via

$\left(S-y_{p}(k)\right)\left(1-\lambda^{H}\right)=y_{m}(k) a_{m}^{H}+K_{m} u(k)\left(1-a_{m}^{H}\right)-y_{m}(k)$,

which can be solved for the manipulated variable $u(k)$, as

$u(k)=\frac{\left(S-y_{p}(k)\right)\left(1-\lambda^{H}\right)-y_{m}(k) a_{m}^{H}+y_{m}(k)}{K_{m}\left(1-a_{m}^{H}\right)}$.

This is the fundamental PFC control equation in its most elementary form [26]. Many processes in production industries can be approximated by a first order system model and, in many PFC control applications, an exponential reference trajectory is used with a single coincidence horizon point $H=1$ and a zero order basis function. Hence the main tuning parameter becomes the desired closed loop response time (CLRT), which is specified by $\tau_{r}$. The controller is tuned in an intuitive manner by adjusting the value of $\tau_{r}$, the desired closed-loop time constant. Although MPC-based controllers can control a system with zero steady state error [27] in the presence of model mismatch, the model will incorrectly estimate the required process input increments at each sample with the result that the closed-loop time constant will not exactly match the desired time constant $\tau_{r}$.

\subsection{Systems with a pure time delay}

For the work described here, the $0.5 \mathrm{~s}$ delay in the VM estimates acts as a pure time delay equivalent to five sample periods in the system. Predictive controllers can take this time delay into account. The delay is not included in the PFC internal process model so that, ideally for a delay of $d$ samples, $y_{p}(k)=y_{m}(k-d)$, and $y_{\text {predict }}(k+d)=y_{p}(k+d)=y_{m}(k)$. Hence, the change in the process output between times $k$ and $k+d$ is equal to the change of the model output between times $k-d$ and $k$, yielding

$y_{p}(k+d)-y_{p}(k)=y_{m}(k)-y_{m}(k-d)$

which rearranges to

$y_{\text {predict }}(k+d)=y_{p}(k+d)=y_{p}(k)+y_{m}(k)-y_{m}(k-d)$.

Hence, the reference trajectory is not initialised on the current of value $y_{m}(k)$, but on the predicted value of $y_{p}(k+d)$ in order to anticipate its response. The control equation given in (10) is still valid by replacing $y_{p}(k)$ with the expression for $y_{\text {predict }}(k+d)$ in $(12)$.
Table 1

Design of experiment inputs for VM model with varying pressure.

\begin{tabular}{lrr}
\hline & Low & High \\
\hline Chamber power $(\mathrm{W})$ & 200 & 600 \\
Ground impedance $(\Omega)$ & 0 & 25 \\
Pressure (mTorr) & 200 & 300 \\
\hline
\end{tabular}

\section{Virtual metrology}

This section describes the steps taken to develop VM models for plasma electron density and plasma etch rate using the PIM data. As discussed in Section 2.2, the VM estimates are delayed by a constant value of $0.5 \mathrm{~s}$ compared to the actual electron density as a result of the operation of the internal circuitry in the PIM sensors.

\subsection{VM of electron density}

The microwave probe is an invasive measurement of plasma electron density that presents a number of disadvantages if used for control:

1. Production wafers cannot be etched while the probe is inserted in the chamber because the plasma is perturbed around the probe body.

2. The sampling frequency of the probe is limited to $2 \mathrm{~Hz}$ due to the time required to download and process the reflected current waveform from the oscilloscope.

As indicated in Section 1, VM models, using measurements from the PIM sensors, are used to estimate the plasma electron density for control purposes. The dynamics of the electron density and etch rate are virtually instantaneous with respect to input power, and, as such, static VM models are employed.

Data is first collected from the system when excited over an experimental range, and, following this, an empirical VM model relating the plasma electron density to the recorded PIM data is constructed. Training and test data sets are collected to create the VM models and then test their generalisation performance, respectively. For model training purposes, the chamber parameters are varied over the ranges specified in Table 1 while accompanying electron density measurements are recorded. As shown in Fig. 6, the applied power is varied from $200 \mathrm{~W}$ to $600 \mathrm{~W}$ in steps of $50 \mathrm{~W}$ for fixed values of ground impedance and pressure. For test data,

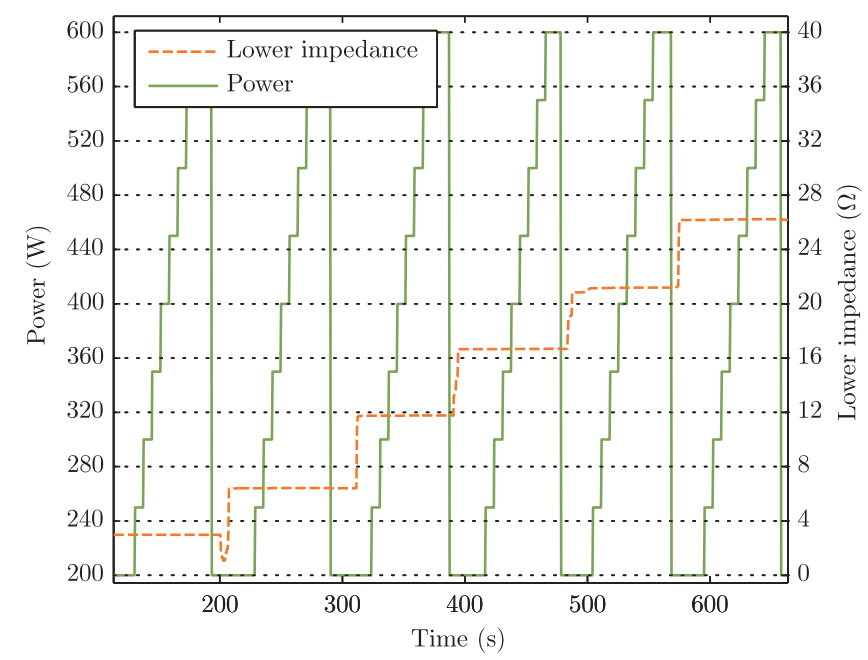

Fig. 6. Designed experiment used for development of electron density VM model showing the system inputs. The inputs shown were repeated at three different pressure set points, 200, 250 and 300 mTorr. 
Table 2

VM estimation results. $R^{2}$ values for all models are greater than 0.99 .

\begin{tabular}{llll}
\hline & $\begin{array}{l}\text { Training MSE } \\
\left(\times 10^{9} \mathrm{~m}^{-3}\right)^{2}\end{array}$ & $\begin{array}{l}\text { Test MSE } \\
\left(\times 10^{9} \mathrm{~m}^{-3}\right)^{2}\end{array}$ & $\begin{array}{l}\text { Max test error } \\
\left(\times 10^{9} \mathrm{~m}^{-3}\right)\end{array}$ \\
\hline MLR & 2.612 & 2.512 & 5.943 \\
ANN & 1.004 & 0.870 & 3.632 \\
GPR & 0.675 & 1.210 & 3.345 \\
\hline
\end{tabular}

the electron density resulting from random values of the system inputs is recorded. The input variables used by the VM models are seven measurements taken from the upper PIM sensor, comprising the fundamental values of the powered electrode current, voltage, phase, and the calculations of the plasma impedance, reactance, resistance, and power.

Three modelling techniques known to produce accurate estimates for plasma etch modelling [4,28], Multiple linear regression (MLR) [29], artificial neural networks (ANNs) [30], and Gaussian process regression (GPR) [31] models, are examined as candidate empirical modelling techniques for VM. The ANNs used have a single hidden layer that is varied in size from one to fifteen neurons and randomly initialised five times during model training. The GPR models use a squared exponential covariance function.

The modelling results are summarised in Table 2, where an ANN model is the most accurate VM model over the unseen test data. MLR models perform worst because the VM input variables are non-linearly related to the electron density over the experimental range. The performances of the ANN and GPR models are quite similar, with the ANN models performing better on unseen test data. Offsets between the estimated and real values of electron density are observed for some system operating points. However, these offsets are rarely greater than $1 \times 10^{9} \mathrm{~cm}^{-3}(\sim 2-3 \%$ absolute error), which is deemed an acceptable level of error for our experimental control work.

\subsection{VM of plasma etch rate}

The procedure for the development of a VM model for plasma etch rate is similar to that used for plasma electron density. Because real-time measurements of plasma etch rate are not available during the etch process, experiments on silicon wafers, where the average etch rate is calculated after processing, are required to map out the operational region for control. Due to operational constraints, the pressure in the etch chamber is kept constant at 300 mTorr during the etch rate VM experiments, a typical production process pressure.

To gather data for VM model creation, 19 wafers were etched in a $\mathrm{He} / \mathrm{SF}_{6}$ plasma at 300 mTorr using different power levels and ground impedance values. The ground impedance was varied from 0 to $25 \Omega$ at powers of 200,300 , and $400 \mathrm{~W}$, etching one wafer for each combination of input values. Etch depth for each wafer was measured after processing, and the corresponding etch rates were calculated. The resulting etch rates are shown in Fig. 7. A further 6 wafers were etched at intermediate settings of power and ground impedance for testing of VM models. As mentioned in Section 2, the thickness of the polysilicon layer on the wafer is measured before and after etching and the average etch rate over the process is calculated.

Along with the measured values for current, voltage, and phase, calculated values of impedance, reactance, resistance, and power are included as input variables to the VM models. Stepwise regression is used to determine the PIM variables that best explain the variance in the etch rate values across the training data set. Current, phase, and power are highlighted as significant variables. Table 3 gives the VM accuracy achieved by the modelling techniques investigated. In this case, due to the large values of etch rate

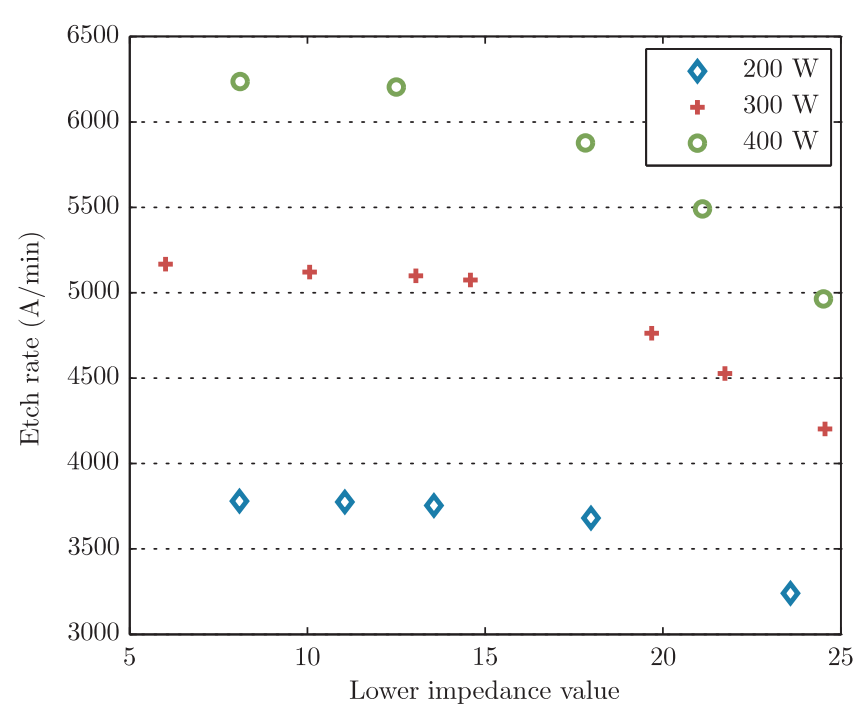

Fig. 7. Average etch rate achieved during data collection for VM modelling.

Table 3

VM estimation results. $R^{2}$ values for all models are greater than 0.99 .

\begin{tabular}{lllc}
\hline & Training MAPE $(\%)$ & Test MAPE $(\%)$ & Max test error $(\AA / \mathrm{min})$ \\
\hline MLR & 0.56 & 0.50 & 55.8 \\
ANN & 0.34 & 0.68 & 68.4 \\
GPR & 0.00 & 1.21 & 122.5 \\
\hline
\end{tabular}

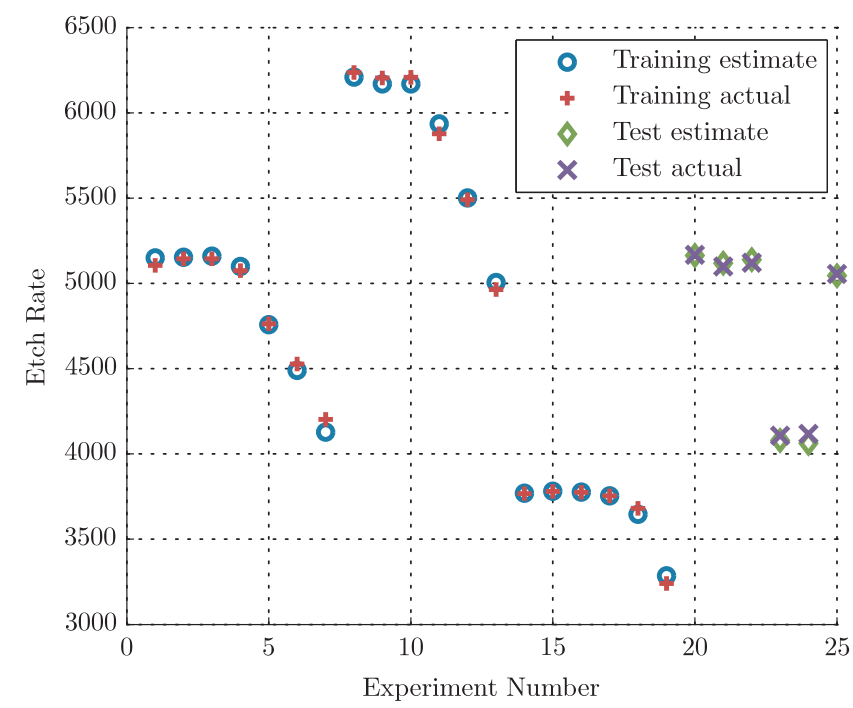

Fig. 8. Etch rate estimates from linear regression model

and correspondingly large errors, MSE metrics greatly exaggerate the errors reported, and so models are compared in terms of mean absolute percentage error (MAPE). An adequate fit for estimation of etch rate was achieved using linear regression models as seen in Fig. 8. The VM model allows non-invasive estimation of etch rate in real time for control purposes.

\section{Control results}

\subsection{Electron density control}

The relationship between the power delivered to the chamber electrode and the plasma electron density is approximately linear for constant values of ground impedance and pressure as 


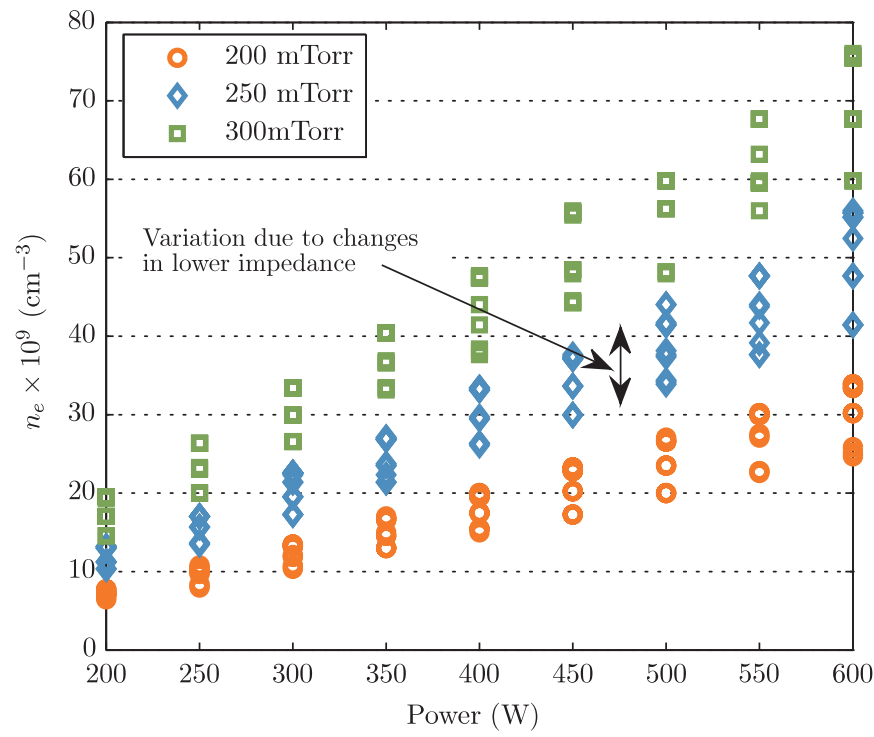

Fig. 9. Electron density response to power at different pressures.

indicated by Fig. 9. Considerable changes in system gain occur when the chamber pressure is changed and for each pressure set point, smaller changes in gain are observed as the ground impedance of the chamber is altered.

Hence, the system, at a specified pressure, can be approximated as a pure gain $K_{m}$, with negligible dynamics and a delay term such that

$G_{m}(s)=K_{m} e^{-\tau_{d} s}$

where $\tau_{d}=d T_{s}$ is the VM delay in seconds. No dynamics are used in this model because the relationship between power and electron density is virtually instantaneous. The lack of dynamics in the system model simplifies the PFC control equations since $a_{m}=e^{-T_{s} / \tau_{m}}=0$ and the system model equation without delay will consist of the forced solution alone. Eq. (10) reduces to

$u(k)=\frac{\left(S-y_{p}(k)\right)\left(1-\lambda^{H}\right)+y_{m}(k)}{K_{m}}$.

Experimental results for set point tracking, and set point tracking in the presence of disturbances in ground impedance, are shown in Figs. 10 and 11, respectively. While the controller sample time is $T_{S}=0.1 \mathrm{~s}$, the electron density is measured separately for validation using the microwave probe at a sampling period of $T_{S}=0.5 \mathrm{~s}$. The ANN VM model accurately estimates the electron density in real time.

For these experiments, the controller is set to produce a conservative closed-loop time constant of $\tau_{r}=1 \mathrm{~s}$. The time constant of $1 \mathrm{~s}$ is chosen as it is a relatively small segment of typical production steps (60-80 s for the etch process run on the tool used), and hence transitions at this rate will not significantly affect final etch results. At a sample time of $T_{s}=0.1 \mathrm{~s}$, ten samples are taken per time constant, allowing reliable control. Additionally, rapid changes in applied power can cause fluctuations in chamber pressure, and the $1 \mathrm{~s}$ response time of the controller ensure that additional anomalies are not introduced to the plasma from the slower pressure control system on the tool. Finally, we observed that much smaller values $(\sim 0.1 \mathrm{~s})$ for $\tau_{r}$ can make the control system sensitive to noise on the VM estimates of electron density, resulting in noisy controlled variables.

Satisfactory control of electron density is achieved at a constant pressure using the real-time VM and PFC control schemes. Set point tracking with time constants of less than $1 \mathrm{~s}$, no overshoot,

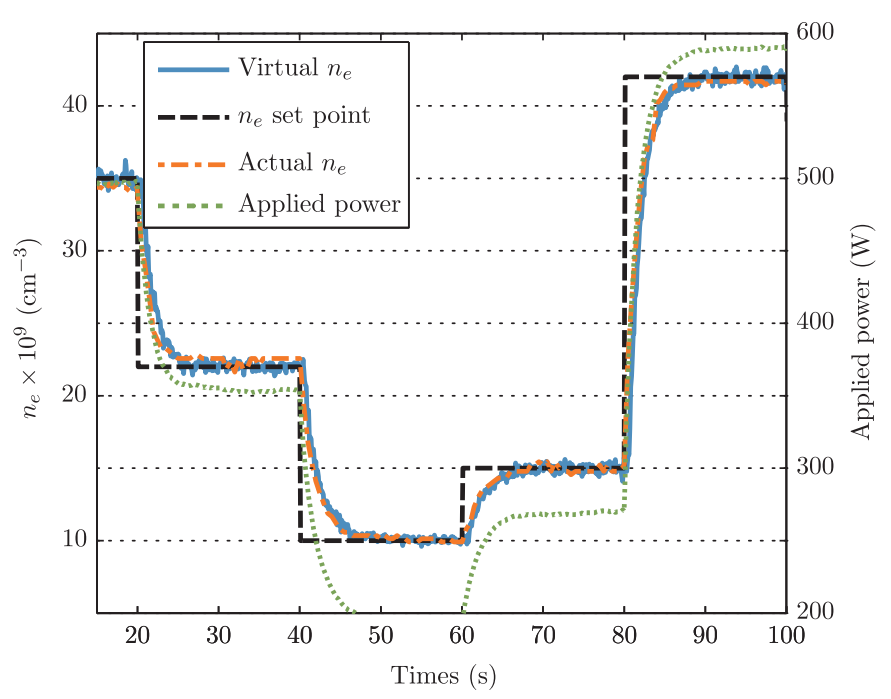

Fig. 10. PFC of electron density with $T_{s}=0.1 \mathrm{~s}, \tau_{r}=1 \mathrm{~s}$, at constant pressure and with no disturbances to the ground impedance (fixed at $4 \Omega$ ).

negligible steady state errors, and robust disturbance rejection properties is demonstrated.

The system gain is not entirely invariant with varying ground impedance or pressure, as shown in Fig. 9. Although the specified closed-loop time constant for the experiments shown in Figs. 10 and 11 is $\tau_{r}=1 \mathrm{~s}$, the system actually responds with a time constant of approximately $1.6 \mathrm{~s}$ due to mismatches between $K_{m}$ and the actual system gain. As described in [32], the PFC internal model gain can be updated continuously using recursive techniques to allow accurate control over larger ranges, compensating for mismatches between $K_{m}$ and the system gain.

\subsection{Plasma etch rate control}

Real-time, in situ measurements of plasma etch rate are unavailable during control experiments. As a result, only the average etch rate over each complete wafer run can be used to validate the control scheme accuracy. Prior to the implementation of the PFC control scheme, a proportional-integral (PI) controller,

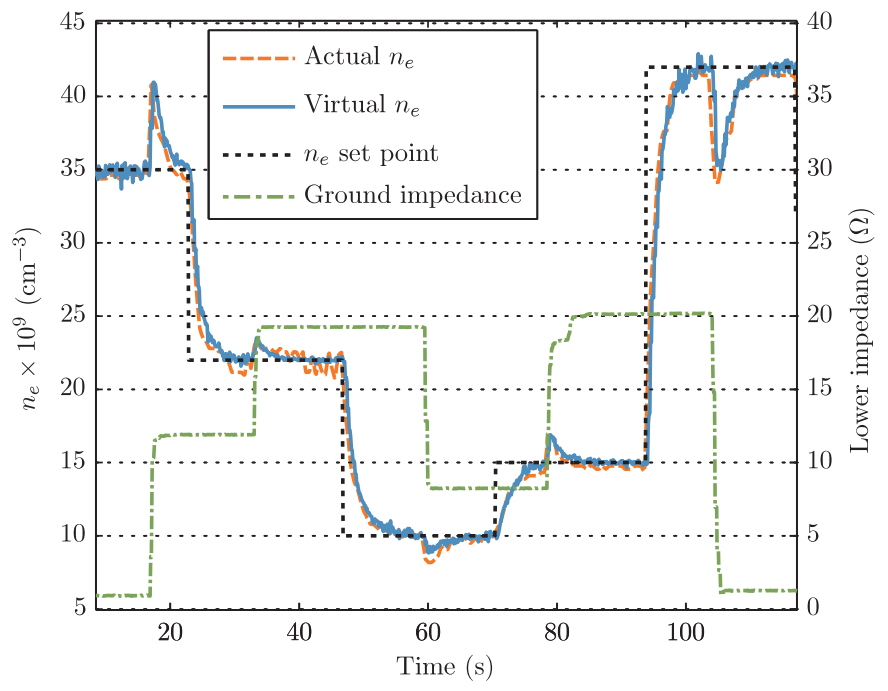

Fig. 11. PFC of electron density with $T_{s}=0.1 \mathrm{~s}, \tau_{r}=1 \mathrm{~s}$, at a constant pressure in the presence of unmeasured disturbances in ground impedance. There is considerable variation in disturbance amplitude since the plasma electron density is more sensitive to disturbances in ground impedance at higher operating powers. 
Table 4

Etch rate (ER) control using PI control with VM system. The MAPE over all experiments was $0.2 \%$.

\begin{tabular}{llll}
\hline ER target $(\AA / \mathrm{min})$ & Ground imp. $(\Omega)$ & ER achieved $(\AA / \mathrm{min})$ & Error $(\%)$ \\
\hline 4500 & 23 & 4511 & 0.2 \\
4500 & 24.8 & 4508 & 0.2 \\
4500 & 13.5 & 4500 & 0.0 \\
4500 & 14.5 & 4488 & 0.3 \\
5500 & 14.5 & 5513 & 0.2 \\
5500 & 16.8 & 5556 & 1.0 \\
5500 & 22 & 5438 & 1.1 \\
5500 & 24.5 & 5362 & 2.5 \\
\hline
\end{tabular}

which facilitates intuitive manual tuning is first investigated as a potential control solution for the plasma etch rate.

Problems with PI control arise from as a result of the $0.5 \mathrm{~s}$ delay caused by the PIM measurements. The delay causes integral windup when the PI controller first starts. In addition, the control is further complicated by the initial transients of the system when the plasma is first ignited. In the initial seconds of the discharge, the molecules in the etchant gases disassociate, resulting in an instantaneous increase in volume and correspondingly, chamber pressure. There is a finite time delay $(\sim 3 \mathrm{~s})$ before the chamber control system counteracts this pressure change to recover the $300 \mathrm{mTorr}$ pressure set point. During this transient period, the controller attempts to compensate for the etch rate values reported by the VM model by increasing the power to the chamber. Hence, to prevent large fluctuations in the applied power, a $2.5 \mathrm{~s}$ delay time is implemented at the start of each control period, during which the applied power is fixed and the controller is disabled.

With this safeguard in place, the PI controller is capable of regulating etch rate based on the real-time VM estimates relatively accurately from the start of the each etch experiment to the end. To test the etch rate control accuracy, wafers are etched with set points in the etch rate that are validated after processing. Unmeasured disturbances are introduced to the ground impedance prior to the etch of each wafer, partially simulating the effect of disruptive PM events. Table 4 gives the etch rate results for PI control for a number of different etch rate targets and ground impedance values. Larger errors are reported for values with high ground impedance values and powers because the plasma starts to change mode in this operational state and the etch rate VM model becomes less accurate.

The startup transients of the chamber are not completely negated by the timed delay during the plasma startup. Examples of the estimated etch rate during plasma ignition are shown in Fig. 12 for two different etch rate set points.

Five wafers were etched with the PFC control scheme to a target etch rate of $3000 \AA / \mathrm{min}$. The PFC control scheme was tuned such that $K_{m}=16$, as determined from the relationship between the applied power and the VM estimate of etch rate, and $\tau_{r}=1 \mathrm{~s}$ to provide a relatively quick response. The ground impedance was varied randomly for each wafer. Table 5 shows the results of this test. As shown in Fig. 13, the PFC control scheme, because it takes the system delay into account explicitly, results in more desirable startup transients than those of the PI controller shown in Fig. 12.

Table 5

Etch rate (ER) control using PI control with VM system. The MAPE over all experiments was $1.36 \%$.

\begin{tabular}{llll}
\hline ER target $(\AA / \mathrm{min})$ & Ground imp. $(\Omega)$ & ER achieved $(\AA / \mathrm{min})$ & Error $(\%)$ \\
\hline 3000 & 8 & 2946 & 1.8 \\
3000 & 12.5 & 2966 & 1.1 \\
3000 & 16.8 & 2993 & 0.2 \\
3000 & 22.1 & 3043 & 1.4 \\
3000 & 24.8 & 3068 & 2.3 \\
\hline
\end{tabular}

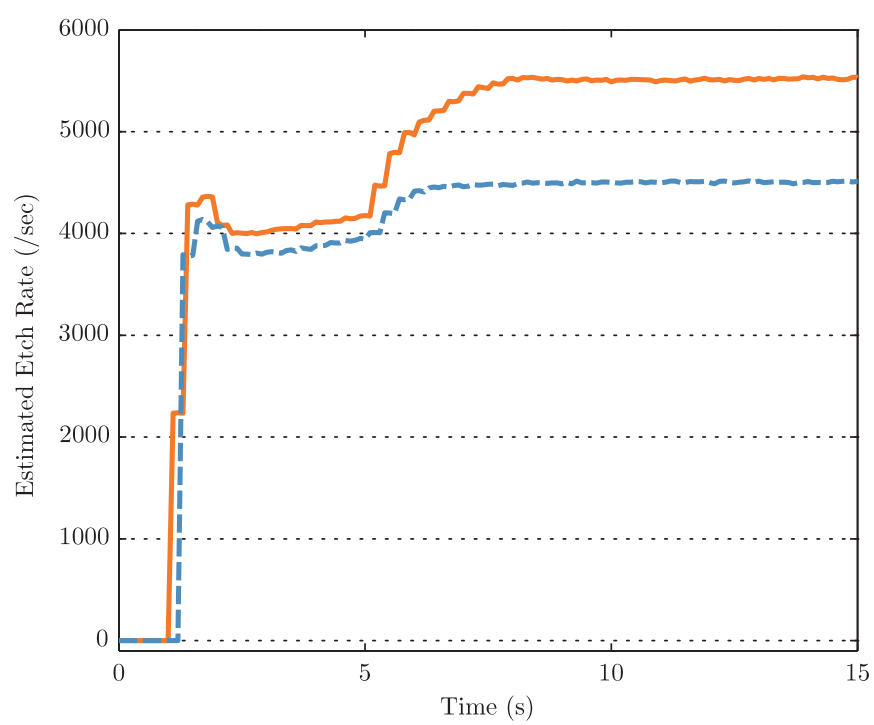

Fig. 12. Startup transients with PI control. Undesirable irregularities are observed as the plasma ignites due to pressure changes in the chamber.

As shown in Fig. 14, the real-time VM and control scheme adjusts the applied power in response to natural within-wafer variance of the chamber conditions to maintain a consistent etch rate. The ground impedance remained constant during this test. Such within-wafer control is advantageous for process reliability when compared to more typical open-loop or run-to-run control schemes where the applied power remains constant for the duration of each wafer etch.

Finally, the real-time VM scheme offers a method to estimate the etch depth during the etch process. Fig. 15 shows the estimated etch rate for a wafer where the etch rate set point has been changed during the wafer run (using PI control). The etch depth reported by the interferometer after the wafer was processed was $4727.1 \AA$. Integrating the $\mathrm{VM}$ etch rate estimate over the experiment duration estimates the etch depth with $0.81 \%$ error at $4689.0 \AA$. Hence, it is feasible to provide a real-time etch depth estimate by integrating the VM etch rate during the etch process.

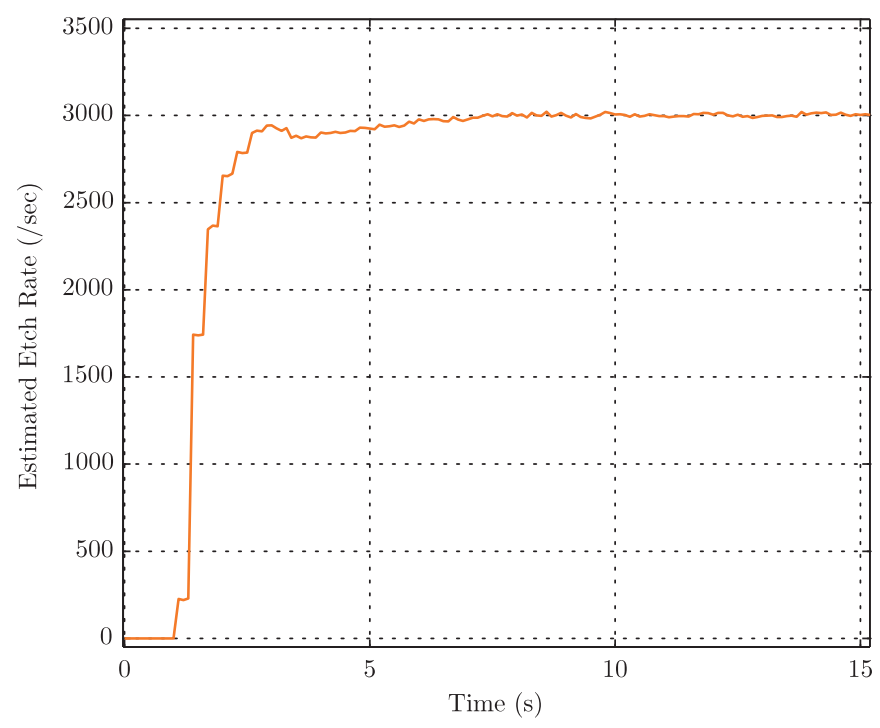

Fig. 13. Startup transients with PFC control. The initial startup irregularities shown in Fig. 12 are much less pronounced because the PFC controller explicitly deals with the system delay in the VM system. 


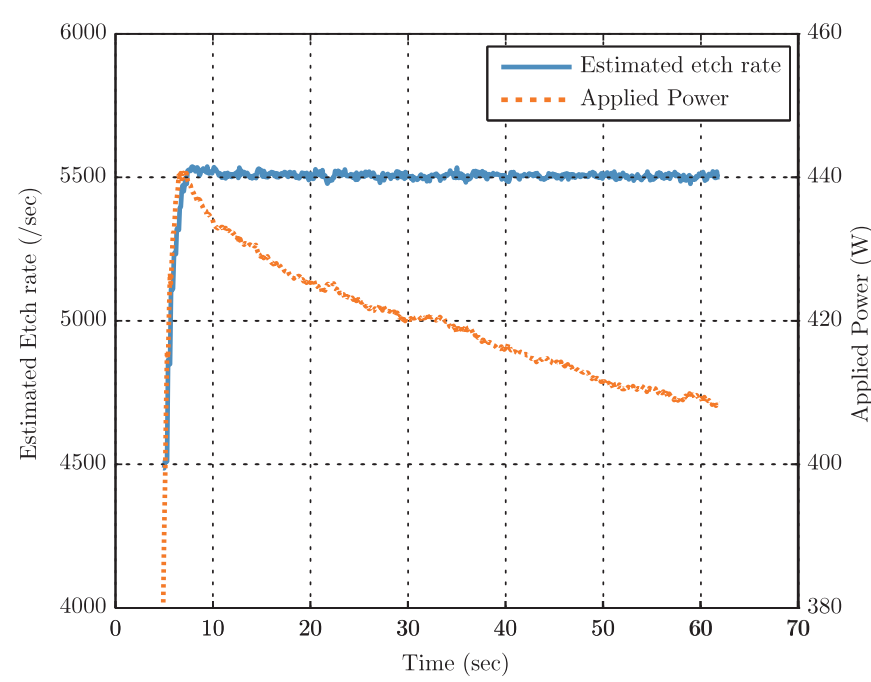

Fig. 14. Real-time adjustment of applied power to maintain a consistent average etch rate in response to within-wafer process variance.

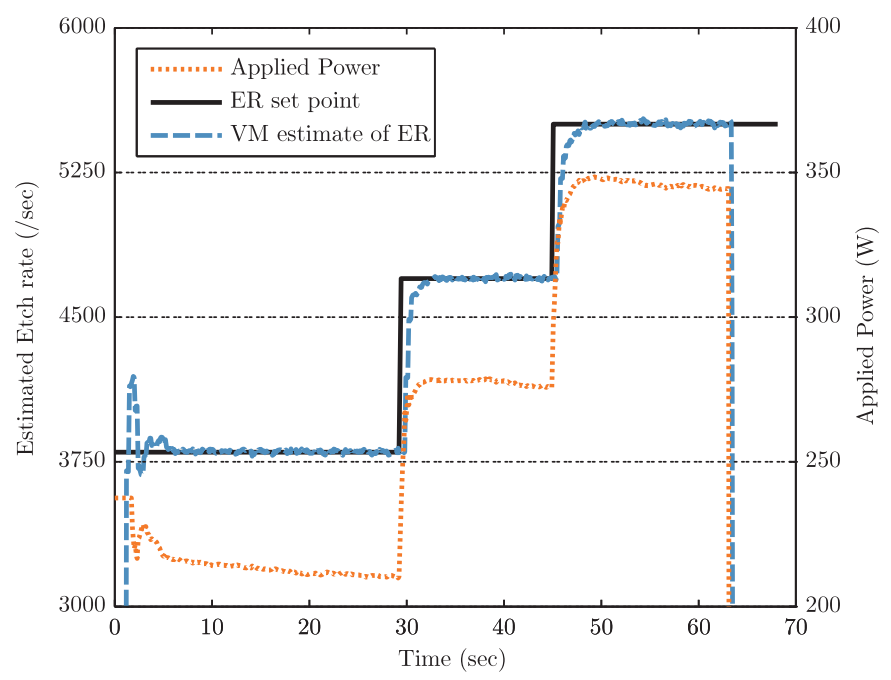

Fig. 15. Estimated etch rate for wafer etch with step changes in etch rate during etching. The etch depth can be estimated with an accuracy of $0.81 \%$ by integrating the VM signal.

\section{Conclusion}

This research has demonstrated the feasibility of real-time VM and control schemes for control of plasma electron density and plasma etch rate in an industrial environment. The use of PIM sensors for VM allows real-time control without perturbation of the processing plasma, and relatively minor modifications to existing chamber hardware.

Electron density set point tracking with time constants of less than $1 \mathrm{~s}$, no overshoot, and negligible steady state errors is demonstrated. Etch rate control, capable of etching wafers to within approximately $1 \%$ of the desired etch rate, using the virtual metrology scheme, is also demonstrated. Through integration of the etch rate estimate, a real-time estimate of etch depth is also available.

The continuous adjustment of process inputs in real-time, during wafer etch, represents a marked improvement upon existing manufacturing techniques, where process inputs are only adapted on a per-wafer or per-lot basis. To migrate the experimental results reported here to a production environment, there are limitations to the technique that require further investigation. Firstly, the VM models for electron density and etch rate may become invalid due to process drift over long durations [33]. When real metrology from drifting processes is available, the VM model will require refreshing to maintain model currency (as proposed in [7,34]). Secondly, separate VM models may be required for different tools in the fabrication environment due to mismatch between tool and sensor characteristics. Model adaptation, rather than complete retraining, may be possible to alleviate the workload of VM model deployment. Finally, further investigation to fully characterise the effects of PM events is required to ensure the VM models can remain accurate.

If implemented successfully, the real-time VM and control schemes have the capability to reduce process variability for plasma etch, allow more complex etching recipes to be realised, and facilitate stricter control limits and hence smaller critical dimensions in future devices. There are also significant economic benefits to improvements in etch feature variance due to potential reductions in the percentage of scrapped wafers and defective products. Also, further increases in throughput can potentially be realised by the potential extension of PM intervals once real-time control of etch features is applied.

\section{Acknowledgements}

The authors would like to thank the staff of Intel Ireland Ltd. for their help with the plasma etch chamber and help with the computer systems of the fabrication environment. The authors would also like to acknowledge the assistance of David Kavanagh from Dublin City University with the hairpin probe measurements of electron density.

\section{References}

[1] G.E. Moore, Cramming more components onto integrated circuits, Electronics 38 (1965) 114-117.

[2] I. Technology Roadmap for Semiconductors, Executive Summary 2009, Technical Report, ITRS, 2009.

[3] F.F. Chen, J.P. Chang, Lecture Notes on Principles of Plasma Processing, Kluwer Academic/Plenum Publishers, 2003.

[4] J.V. Ringwood, S. Lynn, G. Bacelli, B. Ma, E. Ragnoli, S. McLoone, Estimation and control in semiconductor etch: practice and possibilities, IEEE Trans. Semicond. Manuf. 23 (2010) 87-98.

[5] G.S. May, C.J. Spanos, Fundamentals of Semiconductor Manufacturing and Process Control, Wiley-Interscience, 2006.

[6] A. Khan, J. Moyne, D. Tilbury, An approach for factory-wide control utilizing virtual metrology, IEEE Trans. Semicond. Manuf. 20 (2007) 364-375.

[7] A.A. Khan, J. Moyne, D. Tilbury, Virtual metrology and feedback control for semiconductor manufacturing processes using recursive partial least squares, J. Process Control 18 (2008) 961-974.

[8] A. Ferreira, A. Roussy, L. Conde, Virtual metrology models for predicting physical measurement in semiconductor manufacturing, in: IEEE/SEMI Advanced Semiconductor Manufacturing Conference, 2009, pp. 149-154.

[9] T. Edgar, S. Butler, W. Campbell, C. Pfeiffer, C. Bode, S. Hwang, K. Balakrishnan, J. Hahn, Automatic control in microelectronics manufacturing: practices, challenges, and possibilities, Automatica 36 (2000) 1567-1603.

[10] M. Sarfaty, S. Arulkumar, A. Schwarm, J. Paik, Z. Jimin, P. Rong, M. Seamons, H. Li, R. Hung, S. Parikh, Advance process control solutions for semiconductor manufacturing, in: Advanced Semiconductor Manufacturing Conference, 2002, pp. 101-106.

[11] A. Steinbach, Real time plasma etch diagnostics by plasma monitoring system hercules, in: Frontiers in Low Temperature Plasma Diagnostics III, Centre for Research in Plasma Physics, Siemens, 1999.

[12] T. Lin, F. Cheng, W. Wu, C. Kao, A. Ye, F. Chang, NN-based key-variable selection method for enhancing virtual metrology accuracy, IEEE Trans. Semicond. Manuf. 22 (2009) 204-211.

[13] P.I. Klimecky, J.W. Grizzle, F.L. Terry, Compensation for transient chamber wall condition using real-time plasma density feedback control in an inductively coupled plasma etcher, J. Vac. Sci. Technol. A 21 (2003) 706-717.

[14] S. Imai, Virtual metrology for plasma particle in plasma etching equipment, in: Int. Symp. on Semiconductor Manufacturing, 2007, pp. 1-4.

[15] P. Kang, H. Lee, S. Cho, D. Kim, J. Park, C. Park, S. Doh, A virtual metrology system for semiconductor manufacturing, Expert. Syst. Appl. 36 (2009) 12554-12561.

[16] P. Kang, D. Kim, H. Lee, S. Doh, S. Cho, Virtual metrology for run-to-run control in semiconductor manufacturing, Expert Syst. Appl. 38 (2010) 2508-2522.

[17] M. Sarfaty, C. Baum, M. Harper, N. Hershkowitz, J. Shohet, Real-time monitoring and control of plasma etching, Jpn. J. Appl. Phys. 37 (1998) 2381-2387.

[18] D. Stokes, G. May, Real-time control of reactive ion etching using neural networks, IEEE Trans. Semicond. Manuf. 13 (2000) 469-480. 
[19] D. Stokes, G. May, Indirect adaptive control of reactive ion etching using neural networks, IEEE Trans. Robot. Autom. 17 (2001) 650-657.

[20] I. Rosen, T. Parent, B. Fidan, C. Wang, A. Madhukar, Design, development, and testing of real-time feedback controllers for semiconductor etching processe using in situ spectroscopic ellipsometry sensing, IEEE Trans. Control Syst. Technol. 10 (2002) 64-75.

[21] S. Bushman, T.F. Edgar, I. Trachtenberg, Radio frequency diagnostics for plasma etch systems, J. Electrochem. Soc. 144 (1997) 721-732.

22] H.M. Park, C. Garvin, D.S. Grimard, J.W. Grizzle, Control of ion energy in a capacitively coupled reactive ion etcher, J. Electrochem. Soc. 145 (1998) 4247-4252.

[23] R.L. Stenzel, Microwave resonator probe for localized density measurements in weakly magnetized plasmas, Rev. Sci. Instrum. 47 (1976) 603-607.

[24] R.B. Piejak, J. Al-Kuzee, N.S.J. Braithwaite, Hairpin resonator probe measurements in RF plasmas, Plasma Sources Sci. Technol. 14 (2005) 734-743.

[25] R.B. Piejak, V.A. Godyak, R. Garner, B.M. Alexandrovich, N. Sternberg, The hairpin resonator: a plasma density measuring technique revisited, J. Appl. Phys. 95 (2004) 3785-3791.

[26] J. Richalet, D.O. Donovan, Predictive Functional Control, Springer, 2009.
[27] J. Rossiter, J. Richalet, Realigned models for prediction in MPC: a good thing or not? in: Proc. of the 6th Advanced Process Control Conf, 2001, pp. 63-70.

[28] S. Lynn, J. Ringwood, N. MacGearailt, Global and local virtual metrology model for a plasma etch process, IEEE Trans. Semicond. Manuf. 25 (2012) 94-103.

[29] D.C. Montgomery, E.A. Peck, G.G. Vining, Introduction to Linear Regression Analysis, John Wiley \& Sons, Inc., 2001.

[30] S. Haykin, Neural Networks: A Comprehensive Foundation, Prentice Hall, 1999

[31] C.E. Rasmussen, Evaluation of Guassian processes and other methods for nonlinear regression, Ph.D. thesis, Department of Computer Science, University of Toronto, Toronto, ON, Canada, 1996.

[32] S. Lynn, N. MacGearailt, J. Ringwood, Real-time virtual metrology and control of plasma electron density in an industrial plasma etch chamber, in: IFAC World Congress, Milano, Italy, 2011.

[33] D. Tsunami, J. McNames, B. Whitefield, P. Rudolph, J. Zola, Oxide etch rate estimation using plasma impedance monitoring, in: SPIE, vol. 5755, 2005, pp. 59-68.

[34] F. Cheng, H.-C. Huang, C.-A. Kao, Dual-phase virtual metrology scheme IEEE Trans. Semicond. Manuf. 20 (2007) 566-571. 